\title{
Classical Relativistic Particles
}

\author{
R. ARENS \\ University of California, Los Angeles, California
}

Received August 25, 1970

\begin{abstract}
The concept of Lorentz-invariant classical elementary particle is made precise and it is found that there are exactly two families: $(0)$ the well-known free pointparticles distinguished only by their masses and $(+)$ a family with eight-dimensional phase space whose members are distinguished either by their mass or their positive spin.
\end{abstract}

\section{Introduction}

An invariant elementary one-particle system has a space of states $M$ in which the space-time group (here Poincaré, i.e., inhomogeneous Lorentz group) operates or acts in a transitive way. We require transitivity in our definition because if there were a non-trivial subset of states which was invariant then this would define a more elementary system.

We require also that for each state $m$ (or initial condition for some Lorentz frame) there should be defined a position and velocity $\pi(m)$, to be thought of as the state of the centroid. This map $\pi$ should be such that the centroid moves in the way a free point-particle is always supposed to move.

The last requirement is that there should be a Poisson bracket defined for functions on $M$ and "generating" functions $h_{1}, \ldots, h_{10}$ such that the infinitesimal space-time transformations $X_{1}, \ldots, X_{10}$ are represented by $X_{i} f=\left\{h_{i}, f\right\}$.

In the present paper, we classify only the cases where $M$ is connected. The result is that there is allowed not only the free point-particles with various positive masses, but also systems with eight-dimensional state space. These are distinguished by their masses and their positive spin value.

For different values of these parameters the systems are not canonically equivalent. However, those with non-zero spin have general features in common with the example (mass 1 and spin 1, usually called $\frac{1}{2}$ ) already presented in [3, Section 5]. It was also shown that this system is the correspondence-limit of the Dirac system.

In all systems of spin zero, the space $M$ is essentially $\boldsymbol{R}^{6}$ (real cartesian six-space) and the action of $\mathscr{P}$ there is the same for all. The individual particle parameter resides in the Poisson bracket. In the systems of nonzero spin, $M$ is essentially $\boldsymbol{R}^{6} \times S^{2}$ where $S^{2}$ is the set of unit vectors in $\boldsymbol{R}^{3}$. 
Again the action of $\mathscr{P}$ in $M$ is independent of the values of the mass and spin parameters. Again, these reside in the symplectic structure. The spin cannot be told by looking at the Hamiltonian.

The point of the paper is then to prove that this list exhausts the possibilities. There is, for example, no system in which $M$ is ten dimensional, even though the ten-dimensional Lie group $\mathscr{P}$ is an excellent space for $\mathscr{P}$ to act in.

The Lie-group-theoretical investigation needed to establish the existence and classification of such particles was completed in [4], and reference to that paper is indispensable.

\section{Invariant Elementary Particle Systems}

Let us take $\boldsymbol{R}^{3}$ as the configuration space for a particle in the most classical sense. Assuming a second-order dynamics, the space of states (or initial conditions) is $T^{1}\left(\boldsymbol{R}^{3}\right)$, which is to say the space of ("bound") vectors in $\boldsymbol{R}^{3}$. If the dynamics is to be compatible with Einstein-Minkowski space-time structure one will make sure that initial conditions with speed less than 1 never lead to conditions with speed greater than or equal to 1 , and discard all vectors in $T^{1}\left(\boldsymbol{R}^{3}\right)$ except those of length less than 1 , for which set we adopt the symbol $T^{1}\left(R^{3}\right)^{0}$.

We know, of course, how the euclidean group $E(3)$ acts in $\boldsymbol{R}^{3}$. From this action there arises an action in $T^{1}\left(\boldsymbol{R}^{3}\right)$. This latter action preserves the lengths of vectors and hence defines an action of $E(3)$ in $T^{1}\left(\boldsymbol{R}^{3}\right)^{0}$. We shall call this the usual action. Now E(3) corresponds to a certain subgroup of the Poincare group (or inhomogeneous Lorentz group) $\mathscr{P}$ which shall be the space-time group underlying our discussion.

By an invariant system we understand a space $M$ (of "states") in which the space-time group acts [3]. By an elementary (invariant) system we mean one in which this action is transitive on $M$. We impose also a slight regularity condition $[4,3.8]$ and achieve that $M$ is equivalent to the space of cosets $\sigma S$ of a closed subgroup $S$ of $\mathscr{P}$. The action in this coset space $\mathscr{P} / S$ is that $\tau$ in $\mathscr{P}$ sends $\sigma S$ to $\tau \sigma S$.

"Equivalent" has the following meaning here. Let $M$ and $N$ be two spaces in which $\mathscr{P}$ acts. These actions are equivalent if there is a $1: 1$ map of $M$ onto $N$ such that $U(\sigma m)=\sigma U(m)$, and $U$ is called the equivalence.

For a one-particle invariant system we require, besides the action of $\mathscr{P}$ in $M$, also a transitive action of $\mathscr{P}$ in $T^{1}\left(\boldsymbol{R}^{3}\right)^{0}$. In this latter action we require that the action of $E(3)$ be the usual action. Finally we require a mapping $\pi$ from $M$ to $T^{1}\left(\boldsymbol{R}^{3}\right)^{0}$ such that $\pi(\sigma m)=\sigma(\pi(m))$ for all $m$ in $M$ and all $\sigma$ in $\mathscr{P}$. In the right-hand side of this equation we understand the action of $\mathscr{P}$ in $T^{1}\left(\boldsymbol{R}^{3}\right)^{0}$. 
The (real) orthogonal group $O(3)$ lies in $E(3)$ and thus in $\mathscr{P}$. We use $E(1)$ to denote the subgroup of time-translations and time-reversal. The subgroup $E(1) \times O(3)$ of $\mathscr{P}$ is of special interest.

2.1. Theorem. For an elementary invariant one-particle system, the state space $M$ is equivalent to the coset space $\mathscr{P} / S$ where $S$ is some closed subgroup of $E(1) \times O(3)$.

The proof begins with examining the presumed action of $\mathscr{P}$ in $T^{1}\left(\boldsymbol{R}^{3}\right)^{0}$. Let $z$ denote the zero vector at $(000)$ in $\boldsymbol{R}^{3}$. Let $U$ be the subgroup of those $\sigma$ in $\mathscr{P}$ such that $\sigma z=z$. Since $O(3)$ acts in the usual way we see that $O(3)$ lies in $U$. Now we shall show that $E(1)$ does. Let $\sigma_{t}$ be the time-translation by $t$. Then $\sigma_{t} z$ is some vector $z(t)$ in $R^{3}$, say at the point $x(t)$, and with components $v_{1}(t), v_{2}(t), v_{3}(t)$ so that we may write symbolically

$$
\sigma_{t} z=(x(t), v(t)) .
$$

Now let $\varrho$ belong to $O(3)$. Then $\varrho \sigma_{t} z=(\varrho x(t), \varrho v(t))$. But $\varrho \sigma_{t}=\sigma_{t} \varrho$ so $\varrho \sigma_{t} z=\sigma_{t} z$. Thus $\varrho x(t)=x(t)$ and $\varrho v(t)=v(t)$ for all $\varrho$. Hence $x(t)$ and $v(t)$ must be 0 for all $t$. Thus $\sigma_{t} z=z$ and $\sigma_{t}$ belongs to $U$.

Now consider time-reversal, calling it $i$. Let $i z=(x, v)$. The preceeding argument shows $x=v=0$ whence we can now assert $E(1) \times O(3) \subset U$.

Suppose we had an element $\sigma$ of $U$ which is not in $E(1) \times O(3)$. Let $\tau$ be its square. Consider the Lorentz component $\varphi$ of $\tau$. (This is the image of $\tau$ under the map from $\mathscr{P}$ to the Lorentz group.) Then $\varphi$ is in the component of the identity, that is proper and orthochronous. Availing ourselves of the $O(3)$ contained in $U$ we may suppose $\varphi$ has the form

$$
\left[\begin{array}{llll}
1 & & & \\
& 1 & & \\
& & c & s \\
& & s & c
\end{array}\right]=\varphi
$$

where $c^{2}-s^{2}=1$ and the empty spaces are to be filled with zeros. Let $\psi$ be a rotation

$$
\psi=\left[\begin{array}{rrrr}
1 & & & \\
\cos \theta & \sin \theta & \\
-\sin \theta & \cos \theta & \\
& & & 1
\end{array}\right] .
$$

Then $\varrho \varphi \varrho^{-1} \varphi^{-1}$ is the Lorentz component of something in $U$. The reader is asked to compute $d / d \theta$ of this for $\theta=0$ and observe that it is a non-zero infinitesimal Lorentz matrix, unless $\varphi$ is the identity. Hence if $\varphi$ were not the identity then $U$ would have at least one dimension more than that of $E(1) \times O(3)$, i.e., 4. This would make $\operatorname{dim} \mathscr{P} / U<6$. But $\mathscr{P} / U$ 
is equivalent to $T^{1}\left(\boldsymbol{R}^{3}\right)^{0}$ as we required this action to be transitive. Thus $\varphi$ is the identity. As we already have space and time inversion in $U$, we can suppose that the aforementioned $\sigma$ is merely a translation, and thus already in $E(1) \times O(3)$. This contradiction proves $U=E(1) \times O(3)$.

We know $M$ is equivalent to $\mathscr{P} / S$ and $\pi$ is an equivariant map of $M$ onto $\mathscr{P} / U$. It follows from this that some conjugate of $S$ must be a subgroup of $U$. Shifting to a conjugate subgroup gives an equivalent action. Thus 2.1 is proved.

The theory of such elementary particles is therefore completed when the subgroups of $E(1) \times O(3)$ are listed. We list here their connected components, and take only one sample from each class of conjugate subgroups.

One-dimensional Subgroups.

1a: The time-translations, here called $\boldsymbol{R}$.

1b: The rotations in the $x y$ plane, here called $S O(2)$.

1c: Let $\sigma_{t}$ be as above and let $\varrho_{t}$ be a rotation in the $x y$ plane through the angle $t \theta$. Then this subgroup consists of all $\sigma_{t} \varrho_{t}$.

2.2 Two-dimensional Subgroups. There is only one, that generated by $1 \mathrm{a}$ and $1 \mathrm{~b}$. As this subgroup is important, we shall refer to it later.

Three-dimensional Subgroups. There is only SO(3).

The list is completed by the zero-dimensional subgroup, and by $E(1) \times O(3)$ itself, which is four dimensional.

As we shall see later, the odd-dimensional subgroups and the zero subgroup are ruled out by the requirement of Hamiltonicity.

We shall give two examples to show that, conversely, with either the subgroup 2.2 or $E(1) \times O(3)$, elementary one-particle systems are possible.

Consider the usual Minkowski form $\left(x^{1}\right)^{2}+\cdots+\left(x^{3}\right)^{2}-\left(x^{4}\right)^{2}$ in $\boldsymbol{R}^{4}$. Let $\boldsymbol{T}$ be the family of time-like straight lines in $\boldsymbol{R}^{4}$, where a line is timelike if it has direction components $a^{1}, \ldots, a^{4}$ with $\left(a^{4}\right)^{2}>\left(a^{1}\right)^{2}+\cdots+\left(a^{3}\right)^{2}$. The Poincare group, in its action in $\boldsymbol{R}^{4}$, sends time-like lines into timelike lines, i.e., it acts in this six-dimensional space $\boldsymbol{T}$. Moreover, it acts transitively. Now we construct a map $\pi: \boldsymbol{T} \rightarrow T^{1}\left(\boldsymbol{R}^{3}\right)^{0}$. For a line $\lambda$ in $\boldsymbol{T}$, there is a point $\left(a^{1}, a^{2}, a^{3}, 0\right)$ on it. It can be given direction numbers $\left(b^{1}, b^{2}, b^{3}, 1\right)$. We let $\pi(\lambda)$ be the vector in $\boldsymbol{R}^{3}$ at the point $\left(a^{1}, a^{2}, a^{3}\right)$ with cartesian components $\left(b^{1}, b^{2}, b^{3}\right)$. This mapping is in fact $1: 1$ and so we obtain an action of $\mathscr{P}$ in $T^{1}\left(\boldsymbol{R}^{3}\right)^{0}$. The subgroup $S$ leaving the $x^{4}$ (or "time") axis fixed is evidently $E(1) \times O(3)$, so $T$ is equivalent to $\mathscr{P} / E(1) \times O(3)$.

Let $\boldsymbol{L}_{0}$ be the class of light-like straight lines through the origin 0000 , where "light-like" means that direction components $b^{1}, b^{2}, b^{3}, 1$ can be found for which $\left(b^{1}\right)^{2}+\left(b^{2}\right)^{2}+\left(b^{3}\right)^{2}=1$. Thus $\boldsymbol{L}_{0}$ is equivalent to the (two-dimensional) unit sphere in $\boldsymbol{R}^{3}$. Thus $\mathscr{P}$ acts there. 
Of course this yields an action of $\mathscr{P}$ in $\boldsymbol{T} \times \boldsymbol{L}_{0}$. The interesting fact is that it is transitive. Moreover, there is a map $\pi_{2}$ from $\boldsymbol{T} \times \boldsymbol{L}_{0}$ onto $T^{1}\left(\boldsymbol{R}^{3}\right)^{0}$, where indeed $\pi_{2}(\lambda, \mu)=\pi(\lambda)$ where this second $\pi$ is the one mentioned in connection with $\boldsymbol{T}$. This $\pi_{2}$ has the desired equivariance property, for indeed $\pi_{2}(\sigma \lambda, \sigma \mu)=\pi(\sigma \lambda)=\sigma \pi(\lambda)=\sigma \pi_{2}(\lambda, \mu)$. Here the second "=" sign is simply the manner in which $\mathscr{P}$ was made to act in $T^{1}\left(\boldsymbol{R}^{3}\right)^{0}$. Thus we have an elementary one-particle system. Let $\lambda_{0}$ be the $x^{4}$-axis, and let $\mu_{0}$ have components $(0,0,1,1)$. Then the subgroup $S$ of those $\sigma$ such that $\sigma \lambda_{0}=\lambda_{0}$ and $\sigma \mu_{0}=\mu_{0}$ is readily seen to be $E(1) \times O(2)$.

These examples will be referred to as:

\section{3 the standard actions}

involving $\boldsymbol{T}$, or $T^{1}\left(\boldsymbol{R}^{3}\right)^{0}$, or $\boldsymbol{T} \times \boldsymbol{L}_{0}$, etc., as the case may be.

\section{Completely Hamiltonian Systems}

Following [2], we call an invariant system completely hamiltonian if the infinitesimal space-time mappings in $M$ act by means of generating functions, relative to some Poisson bracket. The purely mathematical aspects of this are exposed in [4], where such actions are called hamiltonian actions of the group in question - here $\mathscr{P}$.

It follows from the preceding enumeration of the subgroups of $E(1) \times O(3)$, and from the fact $[4,3.5]$ that $M$ has to be of even dimension, that the only elementary completely hamiltonian one-particle systems would have to be associated with even-dimensional subgroups $S$ of $E(1) \times O(3)$. The component of the identity has therefore to be either

3.0 the identity element of $\mathscr{P}$,

\section{1 the group 2.2, that is, $\boldsymbol{R} \times S O(2)$}

\section{2 the group $\boldsymbol{R} \times S O(3)$.}

If we take the trivial subgroup 3.0, then the quotient space is $\mathscr{P}$ itself and $[4,4.5]$ shows that no completely hamiltonian systems can have $\mathscr{P}$ as a space of states. The same holds for $S$ any closed discrete subgroup.

We pass over 3.1 in order to study first the simpler case of 3.2. To achieve a connected coset space $M$ (see the introduction) we must have $S=E(1) \times O(2)$ and the action is just the standard action in $\boldsymbol{T}$ (or equivalently, in $\left.T^{1}\left(\mathbb{R}^{3}\right)^{0}\right)$. Let $G$ be the connected component of $\mathscr{P}$. Then $G$ acts transitively in this connected $M$, and $[4,4.8]$ may be applied, yielding the following.

3.3 Theorem. There is a one-parameter family of completely hamiltonian one-particle systems with $T^{1}\left(\boldsymbol{R}^{3}\right)^{0}$ as state space and the standard action. 
It will appear that this parameter can be identified with the mass. To do so we introduce co-ordinates $x^{1}, x^{2}, x^{3}, \dot{x}^{1}, \dot{x}^{2}, \dot{x}^{3}$ into $T^{1}\left(\boldsymbol{R}^{3}\right)$ in the well-known way (compare [3]): $x^{i}$ of a vector in $\boldsymbol{R}^{3}$ is the $i$-th cartesian co-ordinate of its base point, and $\dot{x}^{i}$ of a vector is its $i$-th cartesian component.

Consideration of the Lagrangian

$$
-\left[1-\left(\dot{x}^{1}\right)^{2}-\cdots-\left(\dot{x}^{3}\right)^{2}\right]^{\frac{1}{2}}=-(1-\dot{\boldsymbol{x}} \cdot \dot{\boldsymbol{x}})^{\frac{1}{2}}
$$

suggests replacing the $\dot{x}^{i}$ by

$$
p_{i}=\dot{x}^{i}(1-\dot{\boldsymbol{x}} \cdot \dot{\boldsymbol{x}})^{-\frac{1}{2}} .
$$

These co-ordinates $x^{1}, \ldots, x^{3}, p_{1}, \ldots, p_{3}$ have as their domain of definition exactly $T^{1}\left(\boldsymbol{R}^{3}\right)^{0}$ and map it in $1: 1$ fashion onto $\boldsymbol{R}^{6}$.

We consider a 2-form of familiar appearance:

\section{$3.4 d p_{i} \wedge d x^{i} \quad$ (sum from 1 to 3 )}

in $T^{1}\left(\boldsymbol{R}^{3}\right)^{0}$. It is evidently non-singular. It is a fact that

\section{5 the standard action of $G$ in $T^{1}\left(\boldsymbol{R}^{3}\right)^{0}$ preserves this 2 -form.}

The proof of this is laborious. One first expresses the infinitesimal actions $[3,2.72]$ in terms of the $p$ 's (one sets the $A_{i}=0$ ) and verifies that the ten of them, in the order imposed by the list 4.3 (below), have, in the Poisson bracket based on 3.4, generating functions

$3.51(1+\boldsymbol{p} \cdot \boldsymbol{p})^{\frac{1}{2}},-p_{i},-\varepsilon_{i j k} p_{j} x^{k},-x^{i}(1+\boldsymbol{p} \cdot \boldsymbol{p})^{\frac{1}{2}}$.

As observed in $[4$, second sentence after 2.5], this shows that 3.4 is preserved. Hence any constant multiple

$$
3.6 \lambda d p_{i} \wedge d x^{i} \quad(\lambda \neq 0)
$$

is also preserved. This is the parameter promised by 3.3. Actually $[4,4.8]$, whose proof is much easier than the direct attack on 3.4, very readily shows that 3.6 gives the only hamiltonization.

Now when a constant $\lambda$ is introduced, the generating functions get multiplied by the same factor $[4,2.4 .1]$, so now we know what they would be. Of course one can still add arbitrary constants. We adopt the following conventional definition of mass.

Choose those particular "normalized" generating functions (in the abstract notation of $\left[4\right.$, Section 6]) $h_{1}, \ldots, h_{N}$ for which $h_{i}=\left\langle A_{k} \mu^{k} ; Y_{i}\right\rangle$, as we know is possible. (This settles the problem of the arbitrary constants that could have been added.) Then shifting back to the Poincaré group, let $h_{1} h_{2} h_{3} h_{4}$ be those generating the space and time translations 
and define $m \geqq 0$ by ${ }^{1}$

$3.7 m^{2}=h_{4}^{2}-h_{1}^{2}-h_{2}^{2}-h_{3}^{2}$.

How do we know that 3.51 really corresponds to this choice of the additive constants? We test it by seeing if they satisfy the commutation relations exactly, for this is obviously characteristic of the normalized ones. Thus the mass for 3.51 is 1 , and with $3.6,|\lambda|$.

We defined equivalence in Section 2. This prompts the question: can two systems on $T^{1}\left(\boldsymbol{R}^{3}\right)^{0}$ with different masses, be equivalent? This point is not covered in $[4,4.8]$. Mathematically this comes down to the following question: is there a $1: 1$ mapping $U$ of $T^{1}\left(\boldsymbol{R}^{3}\right)^{0}$ onto itself which commutes with the action of $\mathscr{P}$ and transforms $\lambda d p_{i} \wedge d x^{i}$ into $\mu d p_{i} \wedge d x^{i}$ with $|\lambda| \neq|\mu|$ ? The answer is "no". This whole problem becomes more serious for the system involving 3.1 where there are indeed such commuting maps, and we shall prove there that the mass cannot be changed. That discussion can easily be applied to the present case and we leave it to the reader.

\section{The Case $M=T \times L_{0}$}

This is the case 3.1. First we explore it abstractly. According to [4, 4.7], together with the table just preceding that theorem, the Poisson brackets preserved by the standard action in $M=\boldsymbol{T} \times \boldsymbol{L}_{0}$, when $M$ is regarded as a coset space $\mathscr{P} / E(1) \times O(2)$, are those associated with (in the sense $[4,2]$ of being the inverse of) the three-parameter family of 2 -forms $d \mu$ where

$4.1 \mu=-\frac{1}{2}\left(A \mu^{4}+B \mu^{12}+C \mu^{43}\right)$.

Moreover, the determinant of $d \mu$ is essentially $A^{6} B^{2}$.

4.2 Theorem. The standard action which preserves $d \mu$ (with $\mu$ as in 4.1) is equivalent to another action which preserves $d \mu_{0}$ where

$4.21 \mu_{0}=-\frac{1}{2}\left(A \mu^{4}+B \mu^{12}\right)$,

provided $A \neq 0$.

Proof. Let $\tau_{b}$ be the translation in the $x^{3}$ direction by an amount $b$. The right-multiplication

$$
\sigma \rightarrow \sigma \tau_{b}
$$

commutes with the action of $\mathscr{P}$ in $\mathscr{P}$ and also with its action in $\mathscr{P} / \boldsymbol{R} \times S O(2)$ because (as is easily shown) each self-equivalence of the action in $\mathscr{P} / S$ is implemented by right-multiplication by such an element

${ }^{1}$ A dynamical definition of mass for one particle, based only on the action of $\mathscr{P}$ is of course impossible. It is only after a Poisson bracket has been selected that a mass can be defined. 
$\tau$ of $\mathscr{P}$ which makes $\tau^{-1} S \tau=S$, and conversely. We now show that for a suitable $b, \tau_{b}$ transforms $\mu$ into $\mu_{0}$; and this is more than enough to prove 4.2.

The infinitesimal form of right-multiplication by $\tau_{b}$ is the infinitesimal left-invariant vector field $P_{3}$ on $\mathscr{P}$. This changes any left-invariant vector field $X$ to $\left[P_{3}, X\right]=\operatorname{ad} P_{3}(X)$. What it does to the left-invariant differential forms $\mu^{\prime}, \ldots, \mu^{43}$ is given by the transpose of the matrix of ad $P_{3}$. The multiplication table $[4,4.6]$ shows that

$$
\operatorname{ad} P_{3}\left(\begin{array}{l}
M_{12} \\
M_{43} \\
P_{4}
\end{array}\right)=\left(\begin{array}{lll}
0 & 0 & 0 \\
0 & 0 & 1 \\
0 & 0 & 0
\end{array}\right)\left(\begin{array}{l}
M_{12} \\
M_{43} \\
P_{4}
\end{array}\right)
$$

It also shows that $\operatorname{ad} P_{3}$ preserves the linear subspace of $\mathfrak{g}$ spanned by $M_{23} M_{31} M_{41} M_{42} P_{1} P_{2} P_{3}$. It follows that

whence

$$
\left(\operatorname{ad} P_{3}\right) *\left(\begin{array}{l}
\mu^{12} \\
\mu^{43} \\
\mu^{4}
\end{array}\right)=\left(\begin{array}{lll}
0 & 0 & 0 \\
0 & 0 & 0 \\
0 & 1 & 0
\end{array}\right)\left(\begin{array}{l}
\mu^{12} \\
\mu^{43} \\
\mu^{4}
\end{array}\right)
$$

$$
\left(e^{\mathrm{ad} b P_{3}}\right)^{*}\left(\begin{array}{l}
\mu^{12} \\
\mu^{43} \\
\mu^{4}
\end{array}\right)=\left(\begin{array}{lll}
1 & 0 & 0 \\
0 & 1 & 0 \\
0 & b & 1
\end{array}\right)\left(\begin{array}{l}
\mu^{12} \\
\mu^{43} \\
\mu^{4}
\end{array}\right)=\left(\begin{array}{l}
\mu^{12} \\
\mu^{43} \\
\mu^{4}+b \mu^{43}
\end{array}\right)
$$

whence $\exp b P_{3}$ sends $A \mu^{4}+B \mu^{12}+C \mu^{43}$ into $A \mu^{4}+B \mu^{12}+(C+b A) \mu^{43}$. This proves 4.2.

One could proceed along these lines in greater detail to show that an arbitrary self-equivalence could at most change the signs of $A$ and $B$, but this will also follow from another theorem ( 4.4 below) which is needed for another purpose anyway. We want to establish the relation of $A$ to the mass, and of $A$ and $B$ together with the "spin".

We use Definition 3.7. First we observe that $h_{4}^{2}-h_{1}^{2}-h_{2}^{2}-h_{3}^{2}$ is really constant, using $[4,6.2]$. Accordingly we evaluate it at the identity element. Again we use the fact that $h_{i}=\left\langle A_{k} \mu^{k} ; Y_{i}\right\rangle$ whence, at the identity, $h_{i}$ has the value $A_{i}$. The values at the identity, of the generating functions, presented in an order corresponding to the following basis for the Lie algebra

$$
M_{23} M_{31} M_{12} M_{41} M_{42} M_{43} P_{1} P_{2} P_{3} P_{4}
$$

are

\subsection{1}

$00 B 000000 \mathrm{~A}$.

Hence the mass is $|A|$. 
There is also a quartic polynomial $[1,(6)]$ considered to be mass times spin squared. It, too, is constant and 4.31 shows it to be $A^{2} B^{2}$.

Thus $A^{2}$ and $B^{2}$ are related to quantities which do not change under equivalence because generating functions transform covariantly with the infinitesimal transformations to which they are related (see $[4,3.61]$ keeping in mind that the $X$ 's there are what we are calling $Y$ 's here). Also the property of being normalized (see above 3.7) is preserved. We sum this up.

4.4 Theorem. $|A|$ and $|B|$ remain unchanged by every equivalence transformation $U$.

It appears therefore that two completely hamiltonian one-particle systems should be regarded as involving the same "kind" of elementary particle if and only if there is such a map $U$ between their two spaces of states which transforms the one hamiltonian structure (or closed invariant 2-form, or Poisson bracket) into the other. Thus we can say the following.

4.5 A kind of elementary particle is characterized by two numbers: $m$, $s$, where $m>0, s \geqq 0$. If $s=0$ the space of states is $T^{1}\left(\boldsymbol{R}^{3}\right)^{0}$. If $s>0$ the space of states is ${ }^{2} T^{1}\left(\boldsymbol{R}^{3}\right)^{0} \times S^{2}$.

4.5 is evident in view of the earlier parts of this paper.

Another word regarding "kind". Two elementary particle systems and an equivalence $U$ give the diagram

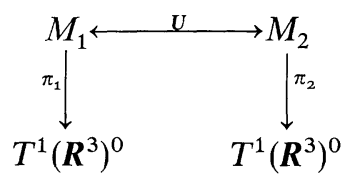

with the requirement $\sigma U\left(m_{1}\right)=U\left(\sigma m_{1}\right)$. We did not require also $\pi_{2}=\pi_{1} \circ U$. In other words, when shifting to a particle of the same kind, $\pi_{1}$ may change. We take the position that an elementary particle system $M$ has to allow at least one action in $T^{1}\left(\boldsymbol{R}^{3}\right)^{0}$ and one $\pi$ as required by the definition, but that these may be altered without changing the elementary particle.

\section{Details in Terms of Coordinates}

In 3.6 we have presented the invariant closed 2-forms for the system with zero spin. The generating functions are those of 3.51 multiplied by $\lambda$.

Just for the record, we shall do the same for the system with spin not zero. The state space we take to be $R^{6} \times S^{2}$ where in $R^{6}$ the cartesian

${ }^{2} S^{2}$ is the unit sphere in $\boldsymbol{R}^{3}$. This is in $1: 1$ correspondence with $\boldsymbol{L}_{0}$. 
coordinates shall be called $x^{1}, x^{2}, x^{3}, p_{1}, p_{2}, p_{3}$. In the 2 -sphere $S^{2}$, functions will be described in terms of three functions $\omega_{1}, \omega_{2}, \omega_{3}$ subject to the condition $\omega_{1}^{2}+\omega_{2}^{2}+\omega_{3}^{2}=1$. A basis for the three-dimensional space of closed invariant 2 -forms is given by $a_{1}, a_{2}, a_{3}$ described as follows:

5.1

Table

$$
\begin{aligned}
& a_{1}=d p_{i} \wedge d x^{i}+\frac{1}{2} \varepsilon^{i j k} \omega_{i} d \omega_{j} \wedge d \omega_{k}, \\
& a_{2}=d p_{i} \wedge d f^{i} \quad \text { where } \quad f^{i}=x^{i}+\frac{(P \times \omega)^{i}}{1+h}, \quad h=(1+p \cdot p)^{\frac{1}{2}}
\end{aligned}
$$

and

$$
a_{3}=d p_{i} \wedge d g^{i} \quad \text { where } \quad g^{i}=\omega^{i}-\frac{p \cdot \omega}{h(1+h)} p_{i} .
$$

For $\alpha=\lambda a_{1}+\mu a_{2}+v a_{3}$, the determinant is $(\lambda+\mu)^{6} \lambda^{2}$, which establishes the connection with 4.1 . We use $(u \times v)^{i}$ to denote the $i$-th component of the cross product.

To assemble the generating functions one takes the $(\lambda, \mu, v)$ linear combinations of the respective functions $a_{i}$-associated with the member of 4.3 in question. We now present these ten associated functions in each case, in order of 4.3. We now relabel this Lie algebra basis, in order, as follows:

$$
J_{1}, J_{2}, J_{3},-K_{1},-K_{2},-K_{3}, P_{1}, P_{2}, P_{3}, P_{4} .
$$

For $a_{1}$ the associated functions are $(i=1,2,3)$

$$
-\omega_{i}-(p \times x)^{i}, x^{i} h-\frac{(p \times \omega)^{i}}{1+h},-p_{i}, h .
$$

For $a_{2}$ (for $f^{i}$ see above),

$$
5.22(f \times p)^{i}, h f^{i},-p_{i}, h .
$$

For $a_{3}$ (for $g^{i}$ see above)

$$
(\omega \times p)^{i},-h g^{i}, 0,0 .
$$

The last 2-form is of course singular (cf. $\mu^{43}$ ).

However difficult it may have been to discover these formulae, the assertions concerning them are rather easy to verify. It would be very difficult to prove, using coordinates, that $a_{1}, \ldots, a_{3}$ give a basis for all such 2-forms. This is where we appeal to the abstract theory.

There is another long calculation whose results may be recorded here. This is the relation of the unique action in $R^{6} \times S^{2}$ given by any nonsingular $\lambda a_{1}+\mu a_{2}+v a_{3}$ with the action in $T^{1}\left(\boldsymbol{R}^{3}\right) \times S^{2}$ obtained from the identification of this with $\boldsymbol{T} \times \boldsymbol{L}_{0}$. These actions are equivalent. It is the expression of this equivalence in coordinates that will be given. 
Just below 3.3 we introduced coordinates $x, \dot{x}$ into $T^{1}\left(\boldsymbol{R}^{3}\right)^{0}$. These had better be called $y, \dot{y}$. (We use a vector notation when we can.) For describing the points of $S^{2}$ we denote the $b^{i}$ of Section 2 by $\theta^{i}$, so $\theta_{1}^{2}+\theta_{2}^{2}$ $+\theta_{3}^{2}=1$. We recall $f$ and $g$ from 5.1.

An equivalence between these actions is given by

$$
y=-f, \quad \dot{y}=\frac{p}{h}, \quad \theta=\frac{p}{h}+\frac{g}{h+p \cdot \omega} .
$$

Relations 5.3 can be discovered as follows. In an action equivalent to either one of these, there are two linear relations between the vector fields representing the elements of 5.2. For example, in $T^{1}\left(R^{3}\right)^{0} \times S^{2}$ we have the relation $P_{4}=-\dot{y} \cdot P$. (The coefficients are not constant.) In $R^{6} \times S^{2}$ we can derive a similar relation from the fact that $h^{2}=1+p \cdot p$. Comparison of coefficients yields $y=p / h$. The other relation originates in the constancy of spin. By comparing its coefficients in the two cases we obtain $y$ and $\theta$.

Now $\pi(x, p ; \omega)=(y, \dot{y})$ is an acceptable projection for the system involved. It is perhaps surprising that $\pi_{1}(x, p, \omega)=(x, \dot{y})$ will not do. The reason it will not is that the vector field for $-K_{i}$ has $x$-components which do not depend only on $x$ and $p$. Hence the projection $\pi_{1}$ does not define a system of imprimitivity for the action of $\mathscr{P}$.

Thus $y$, but not $x$, can be taken as a position observable.

Acknowledgments. This research was supported by NSF grant 18127. The author is grateful to Professor Abdus Salam, the International Atomic Energy Agency and UNESCO at the International Centre for Theoretical Physics, Trieste, where the report was prepared.

\title{
References
}

1. Bargmann, V., Wigner, E. P.: Group-theoretical discussion of relativistic waveequations. Proc. Natl. Acad. Sci. U.S. 34, 211-223 (1948).

2. Babbitt, D. G., Arens, R.: The geometry of relativistic $n$ particle interactions. Pacific J. Math. 28, 243-274 (1969).

3. Arens, R.: A quantum dynamical relativistically invariant rigidbody system. Trans. Am. Math. Soc. 147, 153-201 (1970).

4. - Hamiltonian structures for homogeneous spaces. Commun. math. Phys. 21, 125-138 (1971).

\author{
R. Arens \\ Department of Mathematics \\ University of California \\ 405 Hilgard Avenue \\ Los Angeles, Cal. 90024, USA
}

